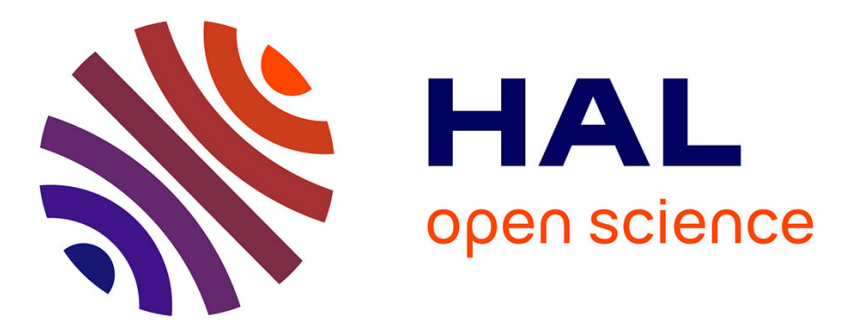

\title{
Analytical coupling losses modelling with COLISEUM: generalized approach upgraded to all stages
}

Maxime Chiletti, Jean-Luc Duchateau, Alexandre Louzguiti, Frédéric Topin, Bernard Turck, Louis Zani

\section{- To cite this version:}

Maxime Chiletti, Jean-Luc Duchateau, Alexandre Louzguiti, Frédéric Topin, Bernard Turck, et al.. Analytical coupling losses modelling with COLISEUM: generalized approach upgraded to all stages. IEEE Transactions on Applied Superconductivity, 2021, 31 (5), pp.5901105. 10.1109/TASC.2021.3063057 . hal-03086189

\section{HAL Id: hal-03086189 https://hal.science/hal-03086189}

Submitted on 21 Feb 2022

HAL is a multi-disciplinary open access archive for the deposit and dissemination of scientific research documents, whether they are published or not. The documents may come from teaching and research institutions in France or abroad, or from public or private research centers.
L'archive ouverte pluridisciplinaire HAL, est destinée au dépôt et à la diffusion de documents scientifiques de niveau recherche, publiés ou non, émanant des établissements d'enseignement et de recherche français ou étrangers, des laboratoires publics ou privés. 


\title{
Analytical coupling losses with COLISEUM: generalized approach upgrade to all stages
}

\author{
Maxime Chiletti, Jean-Luc Duchateau, Alexandre Louzguiti, Frédéric Topin, Bernard Turck, and Louis Zani.
}

\begin{abstract}
Predicting analytically the coupling losses generated in a cable for fusion magnets is still a significant challenge. Difficulties are related to the complex geometry of the system: several multi-strand stages embedded in one another with different twist pitches length, difficulty to model multiplets of strands, including compaction to the final shape. A two-stage analytical geometry based model (COLISEUM) has previously been developed at CEA. We try to extend it to any n-stage cables We detail here an iterative enhancement method to an $n$-stage model . We validated it against experimental data and shown that it is robust enough to fit our measured coupling losses. Finally, this upgraded model can be used to assess coupling losses in fusion nstage cables in a particularly precise way from only geometrical information and analytical tools .
\end{abstract}

Index Terms - AC losses, superconducting, CICCs, fusion magnets

\section{INTRODUCTION}

$\mathrm{C}$ COUPLING losses modelling of CICC for fusion magnets remains a domain of interest as seen in [1],[2], [3], [4], [5] and are often obtained by using numerical codes as in [2] or [6]. We propose here an analytical general method applicable to any $n$-stage cable to reach this objective. In order to establish and develop this n-stage model from the two-stage COLISEUM [7], several input parameters are taken into account at each stage, such as: number of bundles $N$, twist pitches length $l_{p}$, interstage conductances $\sigma$, as well as filamentary zone radius of the basic strand $R_{f}$. We show that the initial two-stage COLISEUM [7] can be derived to obtain a time constant and a shielding coefficient $(n \kappa, \tau)$ for each simulated stage. The model enhancement is realized in two steps: Firstly, the reduction of the two-stage COLISEUM's matrix equation from dimension four to two without loss of information and secondly, we rewrite the system of matrix equations as a unique matrix equation in order to describe any cable with an indefinite number of stages.

Starting from a two-stage interaction in the initial model, we

Manuscript received November 25, 2020; revised February 11, 2021; accepted February 26, 2021. This work was supported in part by ASSYSTEM. (Corresponding author: $M$. Chiletti)

Maxime Chiletti is with Commissariat à l'Energie Atomique et aux Energies Alternatives, CEA/DRF/IRFM, CEA Cadarache 13115 St Paul-Lez-Durance, France (e-mail: maxime.chiletti@cea.fr)

Louis Zani, Bernard Turck, Alexandre Louzguiti and Jean-Luc Duchateau are with Commissariat à l'Energie Atomique et aux Energies Alternatives, CEA/DRF/IRFM, CEA Cadarache 13115 St Paul-Lez-Durance, France.

Frédéric Topin is with Aix Marseille Université, CNRS, IUSTI UMR 7343, 13453, Marseille, France (e-mail: Frederic.topin@univ-amu.fr).

Color versions of one or more figures in this article are available at https://doi.org/10.1109/TASC.2021.3063057.

Digital Object Identifier 10.1109/TASC.3063057 implement first neighbors coupling through the rewriting of systems equations. In the end, we obtain a set of $n$ time constants and $n$ shielding coefficients ( $n$-stage), i.e. one couple of magnetic parameter per stage as in the MPAS model [8]. This newly developed $n$-stage COLISEUM is confronted to MPAS and assessed against the data measured at CEA Cadarache in the JOSEFA facility from a sample representative of JT-60SA TF [9].

\section{SyStem REDUCTION}

The initial development of COLISEUM is fully detailed in [7] along with the complete expressions of the time constants $\tau_{i j}$, and of the amplitudes of current $I$. It was then applied to a twostage system which is described by a $4 \times 4$-matrix equation (1):

$$
\text { (1) } I_{0}+[\tau] \dot{I}_{0}=I_{0_{\text {ext }}} \text { where }[\tau]=\left(\begin{array}{cccc}
\tau_{00} & \tau_{01} & 0 & 0 \\
\tau_{10} & \tau_{11} & \tau_{12} & 0 \\
0 & \tau_{21} & \tau_{22} & \tau_{23} \\
0 & 0 & \tau_{32} & \tau_{33}
\end{array}\right)
$$

After diagonalization of the matrix $[\tau]$, we obtain the four coupled time constants $\tau_{i}$ of the related system (two-stage cable) and evaluate the four corresponding shielding coefficients $n \kappa_{i}$ by using the procedure explained in [7].

We previously showed [6] that among the four magnetic parameters, two $n \kappa_{0} \tau_{0}$ and $n \kappa_{3} \tau_{3}$ are always close to zero and negligible compared to the others. Therefore, the relevant set of magnetic parameters is the pair $\left(\left(n \kappa_{1}, \tau_{1}\right),\left(n \kappa_{2}, \tau_{2}\right)\right)$ where the first set can be related to the first stage contribution and the second set to the second stage one. We also showed that diagonalizing $[\tau]$ in (1) or only its central part by using (3) will give nearly identical pairs of parameters $\left(\left(n \kappa_{1} \tau_{1}\right),\left(n \kappa_{2} \tau_{2}\right)\right)$. This is due to the small norm of the terms $\left(\tau_{00}, \tau_{01}, \tau_{10}, \tau_{32}, \tau_{23}\right.$ and $\left.\tau_{33}\right)$ involved in the diagonalization of the initial matrix $[\tau]$.

The practical most interesting result from dimension reduction is that, we are now able to analytically diagonalize the reduced matrix $[\tau]$ :

$$
[\tau]=\left(\begin{array}{ll}
\tau_{11} & \tau_{12} \\
\tau_{21} & \tau_{22}
\end{array}\right)
$$

where $\tau_{1}$ and $\tau_{2}$ are the eigenvalues of this $2 \times 2$ matrix. Their expressions are:

$$
\begin{aligned}
& \tau_{1}=\frac{1}{2}\left(\tau_{11}+\tau_{22}+\sqrt{\left(\tau_{11}-\tau_{22}\right)^{2}+4 \tau_{12} \tau_{21}}\right) \\
& \tau_{2}=\frac{1}{2}\left(\tau_{11}+\tau_{22}-\sqrt{\left(\tau_{11}-\tau_{22}\right)^{2}+4 \tau_{12} \tau_{21}}\right)
\end{aligned}
$$

where $\tau_{2}$ is always greater than $\tau_{1}$ as all matrix terms are positive and $\tau_{11}<\tau_{22}$. With this first step, we simplified a four 
TABLE I

COLISEUM OUTPUTS

\begin{tabular}{cccc}
\hline \hline \multicolumn{2}{c}{$4 \times 4$ simulation } & \multicolumn{2}{c}{$2 \times 2$ simulation } \\
\hline $\boldsymbol{n} \boldsymbol{\kappa}(\mathbf{a d i m})$ & $\boldsymbol{\tau}(\boldsymbol{m s})$ & $\boldsymbol{n \kappa}$ (adim) & $\boldsymbol{\tau}(\boldsymbol{m s})$ \\
$5.00 .10^{-5}$ & 0.890 & & \\
$9.03 .10^{-2}$ & 1.30 & $0.903 .10^{-2}$ & 1.30 \\
0.923 & 5.16 & 0.923 & 5.16 \\
$4.30 .10^{-6}$ & 20.4 & & \\
\hline
\end{tabular}

Comparison of magnetic parameters given by the $4 \times 4$ simulation and by the $2 \times 2$ analytic calculation.

\section{$\tau$ relative error (adim)}

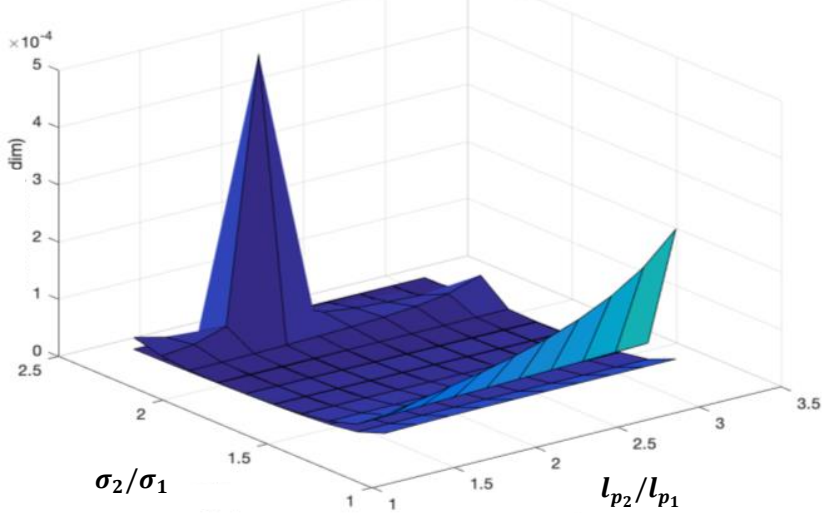

Figure 2: Map of discrepancy between the magnetic parameters $\left(\tau_{1}, \tau_{2}\right)$ given by the four dimensions and the two dimensions system. Shielding coefficients discrepancy exhibits the same behavior.

dimensions matrix equation that must be solved numerically to compute the magnetic parameters of a two-stage cable, to a fully analytical matrix equation of dimension two. After the reduction, we are left with only one couple of magnetic parameters $(n \kappa, \tau)$ per stage as in the MPAS model (see [8]). This reduction has been initiated in [6] with the broad parametric exploration of the two-stage COLISEUM.

Table I show the validation of our simplification in an application case using the geometric parameters of the two-first stage of JT-60SA TF cable that can be found in [9]:

This comparison can be carried out on a large domain of ratio of consecutive twist pitch length ratios $\frac{l_{p_{i+1}}}{l_{p_{i}}}$ and conductances ratios $\frac{\sigma_{i+1}}{\sigma_{i}}$ as shown in Figure 1. We check if the relative error between the four dimensions and two dimensions approach is negligible (as in Table I).

As illustrated on Figure 1 for time constants, we check that the relative difference between the two approaches is negligible on all the explored domain $\left(<5.10^{-2} \%\right)$. These studies on time constants and shielding coefficients are complementary with the study led on the contribution of $n \kappa \tau$ product led in [10]. This show clearly that the dimension reduction does not affect the values of the two remaining time constants and shielding coefficients that are indeed representative of the system. We are now in line with the MPAS formulation of coupling losses: one couple $(n \kappa, \tau)$ per stage of cable (see [8]).

\section{COUPLING AND ITERATION}

Using the reduced two-stage COLISEUM we now extend the model to the description of complex cable with indefinite

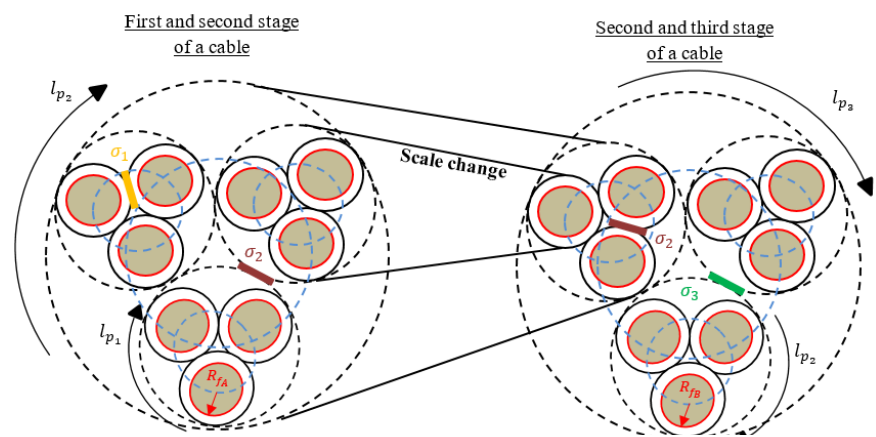

Figure 1: System A on the left, basic elements are $0.81 \mathrm{~mm}$ strands. System B on the right, basic elements are homogenized triplet of strands.

number of stages. We initialize the iteration by considering three consecutive stages that we model by using twice the reduced two-stage COLISEUM: first on system A, a triplet of triplet, i.e. first and second stage of a cable; then, on system B, , also a triplet of triplet, i.e. second and third stage of a cable. We thus try to model three consecutive stages of a cable as depicted in Figure 2.

It should be noted that the second stage of the cable is included in both system: it is the second stage of system A and also the first stage of system B. The two associated model describe respectively the coupling of the first and the second stage in the system $\mathrm{A}$ and the coupling between the second and the third stage in the system B.

System A (cable stage 1 and 2) is described by using:

$$
\left[\begin{array}{l}
I_{1} \\
I_{2}
\end{array}\right]+\left(\begin{array}{cc}
\tau_{11} & \tau_{12} / N_{1} \\
N_{1} \tau_{21} & \tau_{22}
\end{array}\right)_{A}\left[\begin{array}{l}
\dot{I}_{1} \\
\dot{I_{2}}
\end{array}\right]=\left[\begin{array}{l}
4 \sigma_{1} R_{c_{1}} \sin ^{2}\left(\frac{\pi}{N_{1}}\right)\left(\frac{l_{p_{1}}}{2 \pi}\right)^{2} \\
4 \sigma_{2} R_{c_{2}} \sin ^{2}\left(\frac{\pi}{N_{2}}\right)\left(\frac{l_{p_{2}}}{2 \pi}\right)^{2}
\end{array}\right] \dot{B}_{a}
$$

and system B (cable stage 2 and 3 ) is described by using:

$\left[\begin{array}{c}I^{\prime}{ }_{2} \\ I_{3}\end{array}\right]+\left(\begin{array}{cc}\tau_{22} & \tau_{23} / N_{2} \\ N_{2} \tau_{32} & \tau_{33}\end{array}\right)_{B}\left[\begin{array}{c}\dot{I}_{2}^{\prime} \\ \dot{I_{3}}\end{array}\right]=\left[\begin{array}{c}4 \sigma_{2} R_{c_{2}} \sin ^{2}\left(\frac{\pi}{N_{2}}\right)\left(\frac{l_{p_{2}}}{2 \pi}\right)^{2} \\ 4 \sigma_{3} R_{c_{3}} \sin ^{2}\left(\frac{\pi}{N_{3}}\right)\left(\frac{l_{p_{3}}}{2 \pi}\right)^{2}\end{array}\right] \dot{B}_{a}$

It is stressed that $\tau_{22}$ of system $\mathrm{A}$ is strictly equal to $\tau_{22}$ of system $\mathrm{B}$ by construction.

Equation (3.2) is describing the second stage as the super-stage of system A and the equation (4.1) is also describing the second stage, but seen as the sub-stage of system B. In order to get a "full" first neighbor coupling, we couple the two differential equations ((3.2) plus (4.1)) by redefining the coupling between consecutive stages. The new driving equation is:

$N_{1} \tau_{21} \dot{I}_{1}+I_{2}+\tau_{22} \dot{I}_{2}+\frac{\tau_{23}}{N_{2}} \dot{I}_{3}=4 \sigma_{2} R_{c_{2}} \sin ^{2}\left(\frac{\pi}{N_{2}}\right) \dot{B}_{a} / \alpha_{2}^{2}$

Instead having two systems of dimension $2 \times 2$ we can gather both systems into a bigger one of dimension $3 \times 3$ where the second stage will be coupled to first and third stages:

$\left[\begin{array}{l}I_{1} \\ I_{2} \\ I_{3}\end{array}\right]+\left(\begin{array}{ccc}\tau_{11} & \tau_{12} / N_{1} & 0 \\ \tau_{21} N_{1} & \tau_{22} & \tau_{23} / N_{2} \\ 0 & N_{2} \tau_{32} & \tau_{33}\end{array}\right)\left[\begin{array}{l}\dot{I}_{1} \\ \dot{I}_{2} \\ \dot{I}_{3}\end{array}\right]=\left[\begin{array}{l}4 \sigma_{1} R_{c_{1}} \sin ^{2}\left(\frac{\pi}{N_{1}}\right)\left(\frac{l_{p_{1}}}{2 \pi}\right)^{2} \\ 4 \sigma_{2} R_{c_{2}} \sin ^{2}\left(\frac{\pi}{N_{2}}\right)\left(\frac{l_{p_{2}}}{2 \pi}\right)^{2} \\ 4 \sigma_{3} R_{c_{3}} \sin ^{2}\left(\frac{\pi}{N_{3}}\right)\left(\frac{l_{p_{3}}}{2 \pi}\right)^{2}\end{array} \dot{B}_{a}\right.$ 
This new matrix equation of dimension three describes the coupling of three consecutive stages using the first neighbor interaction assumption (mutual inductance). Consistently with MPAS approach, this new system provides one time constant and shielding coefficient per stage (here three stages $=$ three set of magnetic parameters). Iterating this method, we are able to construct and describe any $n$-stage system as presented in [11]. Expression of the matrix equation and magnetic parameters are given in dimension $n$ as well as the dissipated power per unit volume of circumscribed cable. This newly developed model is named $n$-stage COLISEUM.

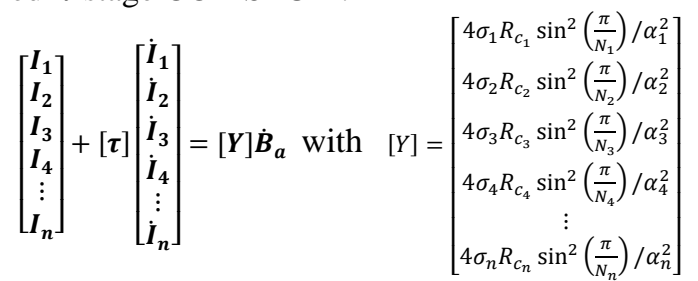

where

$$
[\tau]=\left(\begin{array}{cccccc}
\tau_{11} & \tau_{12} / N_{1} & 0 & 0 & 0 & 0 \\
N_{1} \tau_{21} & \tau_{22} & \tau_{23} / N_{2} & 0 & 0 & 0 \\
0 & N_{2} \tau_{32} & \tau_{33} & \tau_{34} / N_{3} & 0 & 0 \\
0 & 0 & N_{3} \tau_{43} & \tau_{44} & \ddots & 0 \\
0 & 0 & 0 & \ddots & \ddots & \tau_{n-1 n} / N_{n-1} \\
0 & 0 & 0 & 0 & N_{n-1} \tau_{n n-1} & \tau_{n n}
\end{array}\right) \text { (10) }
$$

The coupled time constants $\tau_{i}$ of the system are the diagonal elements of $[\tau]_{\text {coupled }}$ which is obtained by diagonalizing the above time matrix $[\tau]$ by using the passage matrix $[\mathrm{V}]$.

The dissipated power per unit volume of circumscribed cable is expressed by using the same methodology developed in [7]:

$$
P_{v o l}=\lambda_{1} I_{1}^{2}+\lambda_{2} I_{2}^{2}+\cdots+\lambda_{n} I_{n}^{2}
$$

with the $\lambda$ coefficients : $\quad \lambda_{k}=\frac{\left(\frac{l p_{k}}{2 \pi}\right)^{2} \Pi_{l=k}^{n}\left(N_{l}\right)}{8 \sigma_{k} \sin ^{2}\left(\frac{\pi}{N_{k}}\right) \pi R_{c i r c}^{2}}$

The shielding coefficients expressions are extended to the description of $n$-stage cable from those given in [7] as follows:

$$
n \kappa_{i}=2 \mu_{0} \sum_{k=1}^{n} \sum_{l=1}^{n} \lambda_{k} \frac{V_{k i} Y_{b_{i}} V_{k l} Y_{b_{l}}}{\tau_{i}+\tau_{l}}
$$

Where $V_{i j}$ are elements of the passage matrix $[V]$ issued from the diagonilzation of the system and $Y_{b_{i}}$ are elements of the vector $\left[Y_{b}\right]=[V]^{-1}[Y]$.

Coupling losses per cycle of external magnetic field excitation $B_{a}=B_{m} \sin (\omega t)+B_{\text {off }}$ per unit circumscribed volume for such a system writes:

$$
Q_{\text {coupling }}(f)=\sum_{k=1}^{n} n \kappa_{k} \frac{B_{m}^{2}}{\mu_{0}} \frac{\pi \omega \tau_{k}}{1+\left(\omega \tau_{k}\right)^{2}}
$$

\section{CROSSCHECK WITH MPAS AND VALIDATION AGAINST JOSEFA DATA}

\section{A. Application of the n-stage COLISEUM to MAG42-3 data}

We confront the experimental data measured on MAG42-3 samples under transverse sinusoidal field excitation using
TABLE II SET OF CONDUCTANCES

\begin{tabular}{cccccc}
\hline \hline Stage $i$ & 1 & 2 & 3 & 4 & 5 \\
\hline$\sigma_{i}\left(10^{8} S . m^{-1}\right)$ & 0.768 & 0.983 & 1.26 & 1.61 & 2.06 \\
\hline Conductances set chosen for the best fit by using $p=1.28$ and $q=1$. \\
TABLE III \\
n-STAGE COLISEUM OUTPUTS \\
\hline \hline Stage \# & $\boldsymbol{n} \boldsymbol{\kappa}$ (adim) & $\boldsymbol{\tau}$ (ms) \\
\hline 1 & 0.144 & 1.58 \\
3 & 0.177 & 6.67 & \\
4 & 0.425 & 69.3 & \\
5 & 0.193 & & 223 \\
$\boldsymbol{\Sigma n} \boldsymbol{n} \boldsymbol{\tau}$ (ms) & 2.65 & 617 & \\
\hline
\end{tabular}

Magnetic parameters of $n$-stage COLISEUM to fit data from JOSEFA. All shielding coefficients are referred to the area of superconducting strand.

JOSEFA (see [9]) to the modelling given by the $n$-stage COLISEUM. Giving all geometrical parameters of the cable $\left(R_{\text {strand }}, l_{p}\right.$ sequences, and the cable pattern ex: $\left.3 \times 3 \times 3 \times 3 \times 6\right)$ as inputs, it is then possible to construct the $5 \times 5$-matrix equation corresponding to the desired five-stage cable. The only adjustable parameters of the system are the transverse conductances (at each cable stages). We first chose to model the MAG42-3 cable with only five stages without considering the strand interaction with the surrounding stages in order to confront our model to the advanced MPAS (see [9]) which is a five-stage model. Adjusting the conductances set as follows gives us a fairly good agreement with the data we measured in [9] (see Table II and Table III). The conductance set have been restrained and defined by using $\sigma_{1}$ the interstage conductance of the first cabling stage, $p$ the ratio between consecutives $\sigma$ and $q$ a multiplicative factor:

$$
q\left[\begin{array}{lllll}
\sigma_{1} & p \sigma_{1} & p^{2} \sigma_{1} & p^{3} \sigma_{1} & p^{4} \sigma_{1}
\end{array}\right]
$$

The corresponding $Q(f)$ issued from the magnetic parameters of Table III is depicted in Figure 3 along with the experimental coupling losses [9]. We stress that the description of coupling losses given by the $n$-stage COLISEUM in Figure 3 is obtained by hand as a first try of fit for this model (onto JOSEFA's data [9]) and can be improved by the use of a least square method. We also note that the last stage seems to be the major contributor to the predicted coupling losses.

\section{B. Crosscheck with MPAS}

The magnetic parameters from the advanced MPAS gathered in Table IV are slightly different from the one already presented in [9] because this time we use a least square method in order to be sure that the fit found is the best fit.

We can see as already shown before in [9] that the description of coupling losses given by MPAS also point out the fact that the last stage plays a predominant role in coupling losses generated in JT-60SA TF cable. The shielding coefficients given by MPAS are decreasing from the last stage to the first one whereas in $n$-stage COLISEUM the last stage dominates and there is no clear rules for the other sub-stages. Both coupling losses given by the advanced MPAS and the $n$-stage COLISEUM are depicted in Figure 3. 
TABLE IV

MPAS OUTPUTS

\begin{tabular}{|c|c|c|}
\hline Stage \# & $\boldsymbol{n} \boldsymbol{\kappa}$ (adim) & $\boldsymbol{\tau}(\mathrm{ms})$ \\
\hline 1 & 0.220 & 6.02 \\
\hline 2 & 0.254 & 14.6 \\
\hline 3 & 0.293 & 42.8 \\
\hline 4 & 0.340 & 85.9 \\
\hline 5 & 2.23 & 250 \\
\hline $\boldsymbol{\Sigma} \boldsymbol{n} \boldsymbol{\kappa} \boldsymbol{\tau}(\mathrm{ms})$ & \multicolumn{2}{|c|}{604} \\
\hline
\end{tabular}

Magnetic parameters of MPAS to fit data from JOSEFA. All shielding coefficients are referred to the area of superconducting strand.

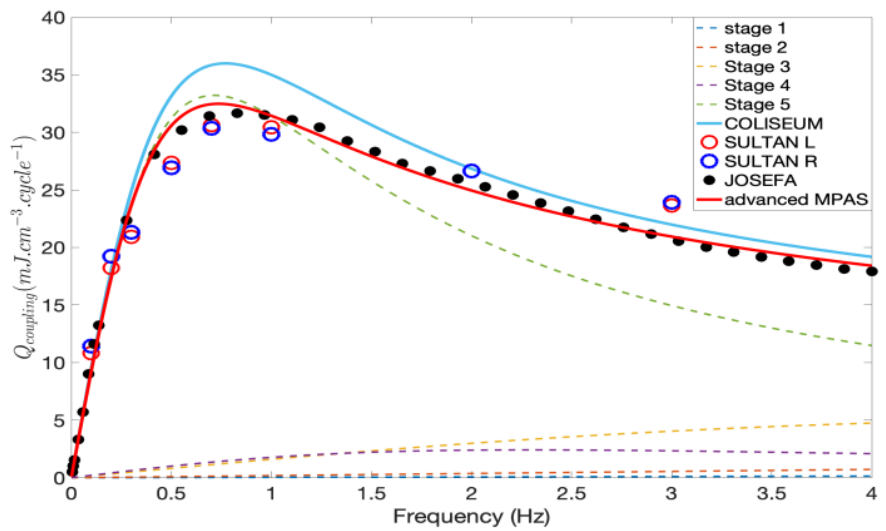

Figure 3: n-stage COLISEUM fit (sky blue) over coupling losses measured in SULTAN and JOSEFA. Only five stages are considered in MPAS (red line) and COLISEUM (blue line). Black dots represent JOSEFA data; Blue and red circles represent SULTAN data. Dashed lines are stages contribution from COLISEUM.

Both descriptions are in good agreement with each other and with the experimental data.

\section{Comments on the n-stage COLISEUM}

We can notice that the behavior shown in [9], i.e. the shift of the coupling losses curves with respect to the void fraction, is perfectly reproducible with the $n$-stage COLISEUM by acting only on conductances set as shown in Figure 4.

We also demonstrate analytically that multiplying globally the whole set of conductances shifts our coupling losses curves toward low or high frequency depending on the multiplying coefficient $q$. The greater conductances are, the lower in frequency the maximum amplitude will be and reversely. If we take $\bar{\tau}_{l}=q \tau_{i}$ as homothetic transformation of all $\sigma$, it will be seen as the change of variable into $q f=F$, giving:

$$
Q(f)=\sum_{i=1}^{j} n \kappa_{i} \frac{B_{m}^{2}}{\mu_{0}} \frac{2 \pi^{2}(f q) \tau_{i}}{1+\left(2 \pi(f q) \tau_{i}\right)^{2}}=\sum_{i=1}^{j} n \kappa_{i} \frac{B_{m}^{2}}{\mu_{0}} \frac{2 \pi^{2} F \tau_{i}}{1+\left(2 \pi F \tau_{i}\right)^{2}}
$$

As in the $n$-stage COLISEUM, time constant are linearly conductance dependent, the effect of a homothetic transformation of the conductance set will be a shift in frequency for the related $Q(f)$ curves (with conserved amplitudes) as seen in Figure 4.

It is established that the $n$-stage COLISEUM can be used to assess the coupling losses of a cable but also to model different void fraction by acting through its conductance set. We note that the behavior obtained after mechanical cycling on CICCs in [12] can be also reproduced as seen in detail in [11].

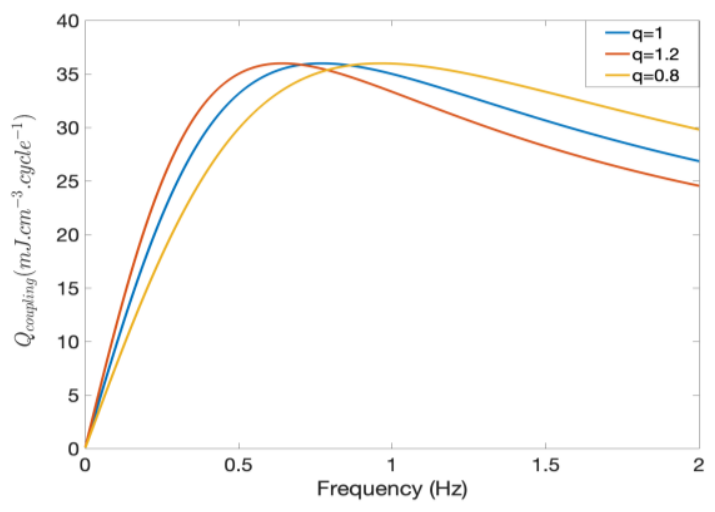

Figure 4: Homothetic transformation of coupling losses by using the $n$-stage COLISEUM. Adjusting the multiplicative parameter $q$ in the conductance set.

\section{CONCLUSION}

In a first step, we succeeded at finalizing the reduction of the two-stage COLISEUM initiated in [7] in order to be in line with the description of coupling losses given by MPAS: one couple of magnetic parameters $(\tau, n \kappa)$ per stage at the twostage level. This was the first step of the iteration of this model to the description of $n$-stage cables.

Then we have established the methodology to iterate the modelling of consecutive cabling stages (see [11] for detailed development). We have presented here the first step of the iteration, i.e. redefining the system equation of consecutive stages in order to introduce the first neighbor interaction.

This redefinition allows to define a matrix equation with dimension equal to the number of simulated stage, allowing COLISEUM to be in line with MPAS description of losses: one couple of magnetic parameter $(\tau, n \kappa)$ per stage for any $n$-stage system. Moreover, the contribution of each stages are compared between MPAS and COLISEUM on one case and we showed that they share a common outcome on coupling losses about the last stage playing a major role in the total of losses.

The newly developed $n$-stage COLISEUM could be used to predict the coupling losses in a CICC but in order to consolidate its predictability, several checks are still to conduct: applying the model on an existing large database of coupling losses tests in order to validate its analytical development and explore its application limits. Crosschecking its prediction ability with existing codes (e.g. JackPot, see [6] [13]) is also an important point.

Further on, the exploration of geometrical compaction cases (deviating the stages from tangency condition) should be explored to apply the $n$-stage COLISEUM in a more realistic cable configuration.

\section{AKNOWLEGMENT}

Authors would like to thank S. Constans and S.Bonici from ASSYSTEM for their financial and technical support, contributing to the realization of the work presented here. 


\section{REFERENCES}

[1] S. Lelekhov. "A physical model and numerical method for losses investigation in superconducting cable-in-conduit conductors (CICC)," Cryogenics v. 46, January, №1, 2006, pp. 1-8.

[2] R. Zanino, M. Bagnasco, F. Bellina, P. Gislon, P.L. Ribani and L. Savoldi Richard, "Modeling AC losses in the ITER NbTi poloidal field full size joint sample (PF-FSJS) using the THELMA code", in Fusion Engineering and Design, Volume 75-79, Pages 23-27, ISSN 0920-3796, 2005.

[3] L. Bottura, P. Bruzzone, J. B. Lister, C. Marinucci and A. Portone, "Computations of AC Loss in the ITER Magnets During Fast Field Transients," in IEEE Transactions on Applied Superconductivity, vol. 17, no. 2, pp. 2438-2441, June 2007, doi: 10.1109/TASC.2007.899837.

[4] E. van Lanen, J. van Nugteren and A. Nijhuis, "Full-scale calculation of the coupling losses in ITER size cable-in-conduit conductors" in Superconductor science and technology, Volume. 25, n², Dec. 2011.

[5] M. Breschi, M. Bianchi, A. C. Ricchiuto, P. L. Ribani and A. Devred, "Analysis of AC Losses in a CS Conductor Sample for the ITER Project," in IEEE Transactions on Applied Superconductivity, vol. 28, no. 3, pp. 1-5, April 2018, Art no. 5900205, doi: 10.1109/TASC.2017.2785404.

[6] E. van Lanen and A. Nijhuis, "JackPot: a novel model to study the influence of current non-uniformity and cabling patterns in cable-in-conduit conductors" Cryogenics, vol. 50, n¹3, pp. 139$148,2010$.

[7] Louzguiti, Alexandre. (2017). Magnetic screening currents and coupling losses induced in superconducting magnets for thermonuclear fusion. $\mathrm{PhD}$ Thesis. CEA Cadarache within DRF/IRFM.

[8] B. Turck and L. Zani, "A macroscopic model for coupling current losses in cables made of multistage of superconducting strands and its experimental validation.", Cryogenics, Volume 50, Issue 8, 2010, Pages 443-449, ISSN 0011-2275.

[9] M. Chiletti, J. Duchateau, F. Topin, B. Turck and L. Zani, " Void fraction influence on CICCs coupling losses: Parametric measurements and analysis with MPAS model," in IEEE Transactions on Applied Superconductivity, October. 2019.

[10] M. Chiletti, J. Duchateau, F. Topin, B. Turck and L. Zani, "Analytical Modelling of CICCs Coupling Losses: Broad Investigation of Two-Stage Model," in IEEE Transactions on Applied Superconductivity, vol. 29, no. 5, pp. 1-5, Aug. 2019, Art no. 4703005. DOI: 10.1109/TASC.2019.2907779.

[11] M. Chiletti (2020) Coupling losses in large superconducting Cable in Conduit Conductors for fusion reactor: Modelling and experimental investigations. PhD Thesis. CEA Cadarache within DRF/IRFM.

[12] Lelekhov S.A., Tronza V.I. "AC Loss before and after Cycling Mechanical Load in ITER RF Cable-in-Conduit Conductor (CICC)". IEEE Transactions on Applied Superconductivity, June 2014, vol. 23, № 3, p. 4201005.

[13] E. van Lanen and A. Nijhuis, "Simulation of Interstrand Coupling Loss in Cable-In-Conduit Conductors With JackPot-AC" in IEEE Transactions on Applied Superconductivity, vol. 21, pp. 19261929, Jul. 2011. DOI:10.1109/TASC.2010.2082474. 